\title{
Research in Serial Port Communication Mechanism Based on Android Mobile
}

\author{
Zhengrong Lei, Xiaoli Long \\ Laboratory Center and Network and Modern \\ Educational Technology Center \\ Guangzhou University \\ Guangzhou,510006,China \\ E-mail: 18938148@qq.com
}

\author{
Waixi Liu \\ School of Electro-Mechanical Engineering \\ Guangzhou University \\ Guangzhou,510006,China \\ E-mail: 18938148@qq.com
}

\begin{abstract}
In this paper, the authors carry out an introduction to the principles of implementing serial port communication by Android mobile and give a detailed explanation to this implementation process through a case. In other words, through .NET serial port communication realizing serial port communication between PC and control equipment, and then Android mobile delivers control signals to the PC serial port by way of Socket communications.
\end{abstract}

Keywords-Android mobile;Serial port;Socket;Control; Equipment

\section{INTRODUCTION}

At present, there are two main ways in controlling equipment: the one way is using PC serial interface (RS-232 or RS-485) to transmit signals to the equipment and control equipment, the two way is using specific interfaces of MCU to connect and control equipment[1].

Because serial port communication technology based on PC is relatively mature, it can provide developers with a simple and easy design development tools and powerful API, developers can easily write serial communication program to realize the control of the device, and now more and more control equipment itself has a serial port or provided by a third-party serial port module. So computer serial port communication technology has become a broader application of the control equipment technology.

Using computer serial port communication technology we can any time read the information of state of the control device to the database in PC through serial port, monitoring equipment and analyzing data.

Android mobile via wifi wireless network can make interconnection to PC, establishing communication mechanisms based on TCP/IP protocol. Android mobile through wifi can send control signals to the PC serial port, and then PC through the serial port send the signal to the control equipment to realize Android mobile controlling equipment, while Android mobile can be connected to the database in PC to extract status information of the control equipment, As shown in Figure 1:

\section{PASSING CONTROL SIGNAL BETWEEN PC SERIAL PORT AND EQUIPMENT SERIAL PORT BY .NET SERIAL PORT COMMUNICATION CONTROLS}

.NET platform is a powerful, collection of a wide variety of programming environments, high efficient application development platform design by Microsoft Corporation. Designers easily realize the specific application development according to their own familiar programming language by calling rich class libraries of .NET platform. To realize serial port communication between PC and equipment in .NET platform, we need only use communication control provided by .NET. Through programming for the corresponding control's properties and events, we can easily implement serial port communications.

Generally there are three methods of programming of serial port communication based on .NET platform: the first way is the application of MSComm control, the easiest and most convenient way, but is required to achieve registration by installing VB6.0 or VC6.0; the second way need not registration, using .NET built-in serial port control directly, we can easily achieve a variety of functions of serial port communication; the third way is to use serial port communications API functions, we can easily achieve the various features, although difficult[1].Following we use serial port control to implement serial port communication, defining two serial port classes PC1_Port and PC2_Port to represent the PC serial port and the equipment serial port.

First of all, define properties of two serial ports and open the corresponding ports:

private void Form1_Load(object sender, EventArgs e) \{

try \{

serialPort1.PortName $=$ comboBox1.Text;

serialPort1. BaudRate $=9600$;

serialPort1.Open();

label2.Text = "the port is visited normally!"; \}

catch (Exception err) \{

label2.Text $=$ "the port does not exist or refuse to visit,please try again!";

\}

\}

Then, define sending data event textBox1_TextChanged at the send port: 


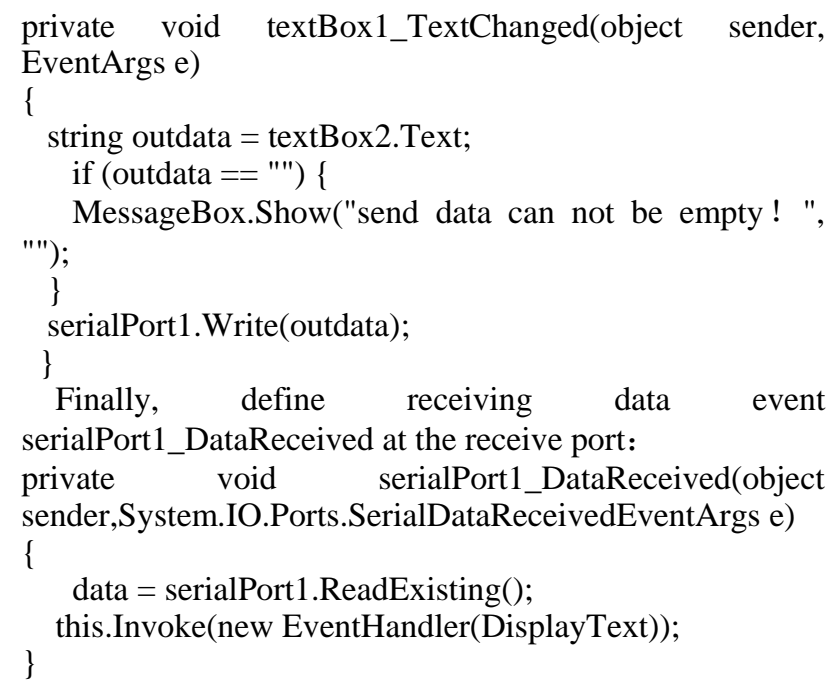

\section{ANDROID MOBILE SEND CONTROL SIGNALS TO THE PC SERIAL PORT IN SOCKET COMMUNICATION WAYS}

Socket is a kind of communication mechanism based on TCP/IP protocol in computer network communication. The application sends and receives data through a socket, like open a file handle for the application, to read and write data to stable storage [2].By socket applications can be added to the network, and communicates with the other applications which are in the same network [2].The information written by the client program on one computer to the socket is capable of being read by service application on another computer, whereas still. Depending on the underlying protocol implementation, socket can be divided into two kinds: the one is based on TCP and the other is based on UDP[3].

Android is a mobile system based on Java platform, full support socket communication mechanisms based on TCP and UDP for JDK environments. Because .NET platform not only with advantages in serial port communications not available in the Java platform, but also with powerful features on the socket communication mechanism. We choose Android as a socket client, and .NET platform as a socket server to establish communication between the Android mobile and the PC, achieving Android mobile to send control signals to PC serial port.

First of all, define an event of receiving Android client data on the PC .NET server:

void bgWorker_DoWork(object sender, DoWorkEventArgs e)

\{

serverSocket $=$ new Socket(AddressFamily.InterNetwork,

SocketType.Stream, ProtocolType.Tcp);

serverIP = IPAddress.Parse("192.168.0.101");

endpoint = new IPEndPoint $(0,30000)$;

serverSocket.Bind(endpoint);

serverSocket.Listen(10);

try \{

while (true) \{ client = serverSocket.Accept();

if (client.Connected) \{

SetLabel("connect Android client successfully!."); \}

else \{

SetLabel("fail to connect Android client！ "); \}

byte[] buffer = new byte[client.ReceiveBufferSize];

if (client.Receive(buffer) $>0$ ) \{

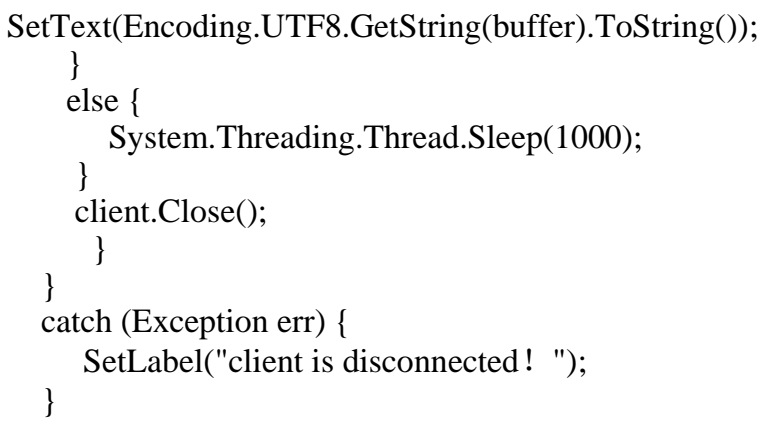

Then, define a button-click trigger event to send data to the PC on the Android mobile client:

sButton.setOnClickListener(new OnClickListener() \{ public void onClick(View v) \{

try \{

\section{SERVERPORT);} mSocket=new Socket(SERVERIP,

String message $=$ show.getText().toString(); out=new PrintWriter(new BufferedWriter(new OutputStreamWriter(mSocket.getOutputStream())),true); out.println(message); out.close(); \} mSocket.close();

catch (Exception e) \{ \}

Log.e("MainActivity", e.toString()); \}

\});

Results as shown in Figure 2:

IV. APPLICATION PROSPECT OF SERIAL PORT COMMUNICATION BASED ON ANDROID MOBILES

Smart phone now has increasingly become the preferred communication tool, in addition to phone calls and text messages for daily function, its biggest advantage is it can run a variety of games and applications as PC, added a endless pleasure to people's lives. Android mobile system is a kind of open source platforms, anyone can download the free Android SDK tools to develop applications based on Android mobiles, while the Android mobile has operation simple, portable, mobile Internet advantages not available in the PC. Believe that in the near future, application research in realizing serial port communication based on Android mobiles will play an important role in the area of the coming 
industrial automation and control, Internet of things, smart home, intelligent robot.

\section{REFERENCES}

[1] Li Jiangquan and Liu Qiao,Visual C\#.NET Serial port communication and its application in measurement and control NET typical examples,Publishing House of Electronics Industry,2012.
[2] Guo Hongzi,Android application development detailed solution.Publishing House of Electronics Industry,2010.

[3] Li Gang,Crazy Android handout.Publishing House of Electronics Industry,2012.

Figure 1. the database in PC to extract status information of the control equipment

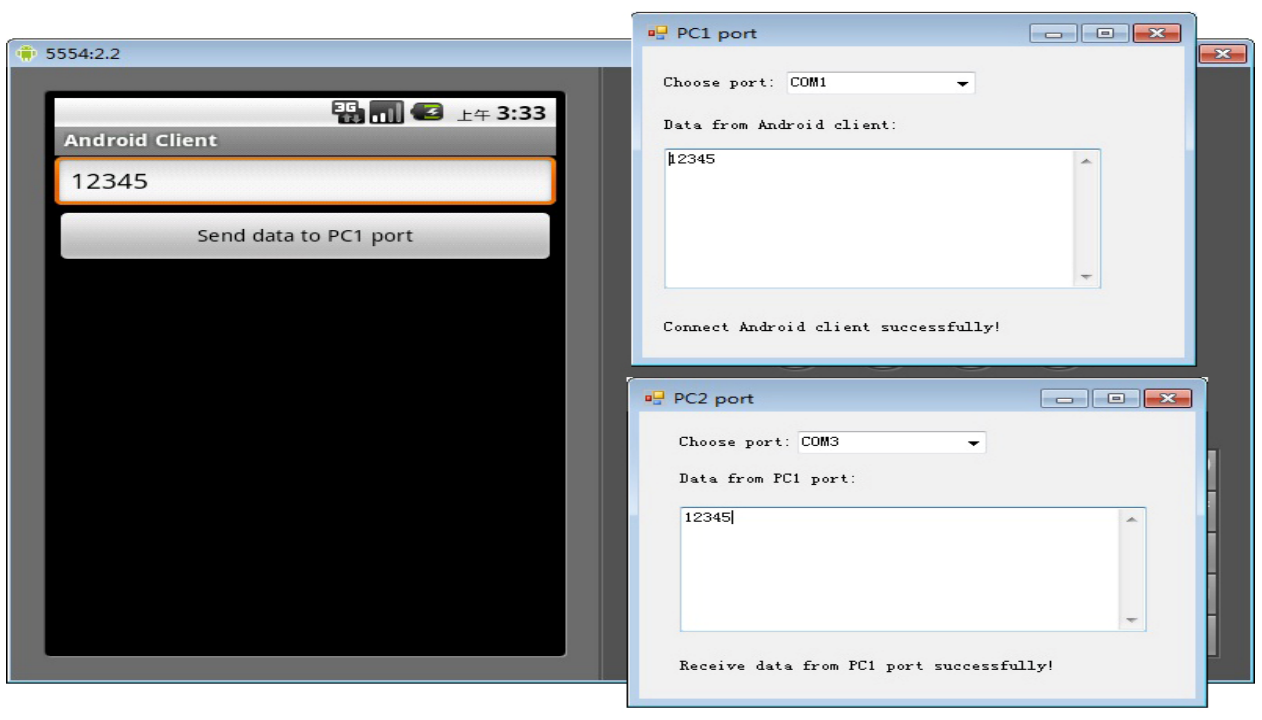

Figure 2. Results as shown 